\title{
Lensing on Cosmic Microwave Background and its Correlation with Large-Scale Structure Surveys
}

\author{
F. Bernardeau \\ Service de Physique Théorique, CEA/DSM/SPhT, Unité de recherche associée au CNRS, \\ CEA/Saclay 91191 Gif-sur-Yvette cédex
}

\begin{abstract}
Lenses acting on the Cosmic Microwave Background can potentially be a very efficient and robust probe of the large-scale matter distribution in the Universe. Their most immediate signature is the way they affect the statistical properties of both the temperature and the polarization fields, in particular they both induce non-Gaussian properties that can be explicitly computed.

Investigations of those effects led to the elaboration of specific reconstruction procedures that aim at mapping the projected mass responsible of the lens effects. These techniques are now on solid ground and are remarkably efficient. However it is likely that our best chance to detect a lensing signal in CMB data in the coming years will be through the cross-correlation patterns they induce between CMB data and tracers of the large-scale structure. Clearly the detection of such an effect would be very fruitful in scrutinizing the gravitational instability picture.
\end{abstract}

\section{Introduction}

Effects of gravitational lenses on background objects are very general and with the advent of deep imaging possibilities are now almost common place in observational cosmology. Their effects on CMB anisotropies are however specific because they make intervene quite a specific source plane. Compared to a galaxy source plane the former has the double advantage of being at a well defined redshift, of being rather thin and having specific patterns, e.g. patterns with well defined statistical properties. For these reasons it clearly makes the phenomenology of gravitational lenses on CMB worth detailed investigations.

From a point of view of the physics of the Cosmic Microwave Background (CMB), lensing effect is one of many secondary effects that can alter the temperature anisotropies of the CMB sky. Among others one could mention effects like the Sunyaev Zel'dovich effect (secondary scattering of photons on hot plasma in galaxy cluster potential wells) or the integrated Sachs Wolfe effect (the observed temperature can be changed if photons travel in time dependent potential wells because of gravitational Doppler effects). Lensing effects have however been recognized as one of the dominant secondary effects (at subdegree scales) because of the large lever arm it takes advantage of (Seljak 1996, Bernardeau 1997, Zaldarriaga and Seljak 1998).

Different aspects of CMB lenses can be investigated. One is obviously the elaboration of best suited strategies for its detection in CMB observations whether it is on CMB temperature maps or on polarization maps. More ambitious is the development of reconstruction methods that would allow the construction of convergence maps out of CMB data. The first generation of $\mathrm{CMB}$ data is however more likely to be better suited for detection of lens effects through CMB-LSS correlations, that is the search of a cross-correlation signal between CMB data and local cosmic shear surveys, the two being likely to share a fair amount of lenses. 


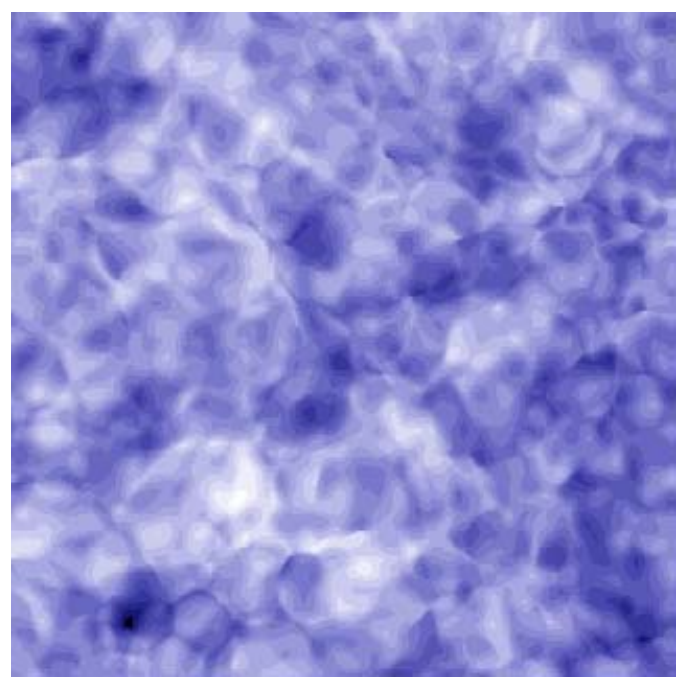

Figure 1. Lens effect on the temperature field. The displacement field has been multiplied by a factor about 5 to make the effects of mode couplings visible.

In this paper these different aspects are reviewed. Section 2 is devoted to the presentation of the basics of CMB lensing. Phenomenological consequences are then explored in Section 3 for the Non-Gaussian properties they induce on CMB temperature maps, in Section 4 for the presentation of reconstruction methods. Finally Section 5 is devoted to the searches that have been undertaken for characterizing the CMB-LSS correlation signals.

\section{Lensing effects on temperature and polarization components}

Gravitational lenses induce bending of the light path, thus moving the apparent position of a sky patch on the last scattering surface by a given angle. The temperature of this patch is not affected itself, i.e. lenses do not created new structures, and a perfectly isotropic sky would remain so. The temperature observed $\vec{\gamma}^{\text {obs. }}$ is thus actually that of position $\vec{\gamma}^{\text {prim. }}$ with,

$$
\vec{\gamma}^{\text {prim. }}(\vec{\gamma})-\vec{\gamma}^{\text {obs. }}(\vec{\gamma})=\frac{2}{c^{2}} \int_{0}^{\chi_{\mathrm{CMB}}} \mathrm{d} \chi \frac{\mathcal{D}_{0}\left(\chi_{\mathrm{CMB}}, \chi\right)}{\mathcal{D}_{0}\left(\chi_{\mathrm{CMB}}\right)} \nabla_{\perp} \phi(\chi),
$$

where $\phi$ of the gravitational potential, $\mathcal{D}_{0}$ is the angular distance, $\chi$ is the distance of the lenses along the line of sight and $\chi_{\mathrm{CMB}}$ is the distance of the last scattering surface (see Mellier 1999).

The displacement affects in a similar manner the temperature and the polarization components. In the following we investigate the consequences of such transforms applied to the CMB observations.

\subsection{On the temperature field}

The local observed temperature field can be expanded in terms of the displacement field which, except for very small scales, is much smaller than the sizes of the temperature patches. It then gives,

$$
\hat{T}(\vec{\gamma})=T(\vec{\gamma})+T_{, i}(\vec{\gamma}) \xi_{i}+\frac{1}{2} T_{, i j}(\vec{\gamma}) \xi_{i} \xi_{j}+\ldots
$$




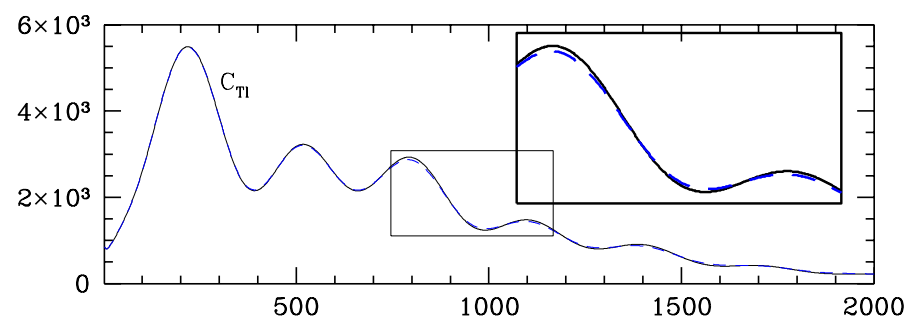

Figure 2. Lens effect on the temperature power spectrum. The insert in the diagram shows the details of the effect of lenses on the temperature power spectrum for the amplitude of the third and fourth peaks.

where $\hat{T}$ is the observed temperature field, $T_{, i}$, its gradient in the direction $i$ (small angle approximation is assumed throughout the paper) and $\xi_{i}$ is the displacement field. This leads to a transform of the different modes of the temperature field. For a full sky survey those modes correspond to the coefficients of the spherical harmonics appearing in such a decomposition. In the CMB literature they are usually denoted $a_{l m}$. In the small angle approximation however, the $a_{l m}$ are simply the Fourier modes of the temperature field $T(\mathbf{l})$. The Fourier modes of the displacement field can be expressed in those of the convergence field $\kappa$. They read $\xi_{i}(\mathbf{l})=-i \mathbf{l}_{i} / l^{2} \kappa(\mathbf{l})$. The relation (2.2) can be easily transposed in Fourier space in,

$$
\hat{T}(\mathbf{l})=T(\mathbf{l})-\frac{\mathbf{l}_{1} \cdot \mathbf{l}_{2}}{l_{2}^{2}} T\left(\mathbf{l}_{1}\right) * \kappa\left(\mathbf{l}_{2}\right)+\frac{1}{2} \frac{\left(\mathbf{l}_{1} \cdot \mathbf{l}_{2}\right)\left(\mathbf{l}_{1} \cdot \mathbf{l}_{3}\right)}{l_{2}^{2} l_{3}^{2}} T\left(\mathbf{l}_{1}\right) * \xi_{i}\left(\mathbf{l}_{2}\right) * \xi_{j}\left(\mathbf{l}_{3}\right)+\ldots
$$

where the second and third terms of the r.h.s. of this equation should be understood as integrals over the modes $\mathbf{l}_{i}$ with the constraint $\sum_{i} \mathbf{l}_{i}=\mathbf{l}$. The most immediate consequence of this expansion is a change of the power spectrum of the temperature field. Indeed when one computes the ensemble average of $\hat{T}(\mathbf{l}) \hat{T}\left(\mathbf{l}^{\prime}\right)$ one has,

$$
\left\langle\hat{T}(\mathbf{l}) \hat{T}\left(\mathbf{l}^{\prime}\right)\right\rangle=\delta_{\text {Dirac }}\left(\mathbf{l}+\mathbf{l}^{\prime}\right) C^{\hat{T}}(l) .
$$

The relation (2.3) leads to the relation,

$$
C^{\hat{T}}(l)=C^{T}(l)\left(1-\frac{l^{2}}{2} \sigma_{\xi}^{2}\right)+\frac{\left(\mathbf{l}_{1} \cdot \mathbf{l}_{2}\right)^{2}}{l_{2}^{4}} C^{T}\left(l_{1}\right) * P^{\kappa}\left(l_{2}\right)
$$

where $\sigma_{\xi}^{2}$ is the variance of the displacement field, $P^{\kappa}(l)$ is the power spectrum of the convergence field and again the second term of the r.h.s. should be understood as a convolution: the power spectrum of the observed temperature field is then a convolution of the one of the primordial anisotropies with the one of the displacement field. It is eventually not dramatically changed. A detailed examination of the results shows that its oscillatory features appear to be slightly damped (see Fig. 2 taken from Seljak 1996).

If the effect of lenses are not dramatic for the power spectrum, they are important in changing the statistical properties of the temperature field. They induce in particular mode couplings effects (they are somewhat visible on the numerical experiment shown on Fig. 1) that induce non-Gaussian properties in the temperature field. This will be explored in the next section.

\subsection{On the polarization field}

The lens effect affects the local polarization just by moving the apparent direction of the line of sight as shown by Faraoni (1993). Thus, if we use the Stokes parameters $Q$ 

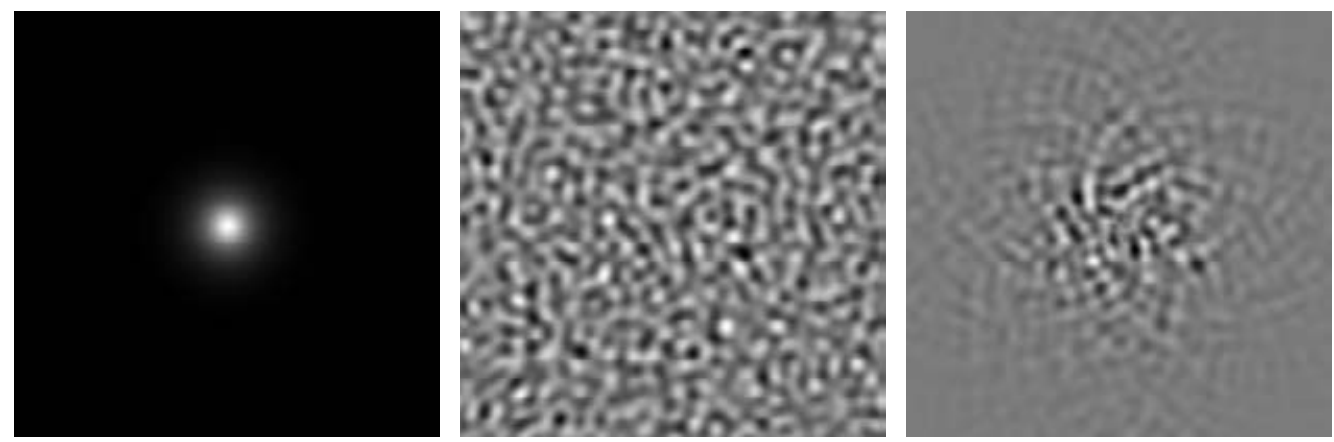

Figure 3. Lens effect induced by a large isothermal sphere with finite core radius. The $\kappa$ map of the lens is shown on left panel. The primordial $E$ sky is presented in the middle left panel. It has been generated for a $\Omega_{0}=0.3, \Lambda=0.7$ model, without tensor modes. The right panel displays the true reconstructed $\Delta \hat{B}$ field in a $4.5 \times 4.5$ degree map (from Benabed et al. 2001 .

and $U$ to describe the local polarization vector, we can relate the observed polarization components $\hat{Q}$ and $\hat{U}$ to the primordial one by the relation

$$
\hat{Q}(\vec{\alpha})=Q(\vec{\alpha}+\vec{\xi}), \quad \hat{U}(\vec{\alpha})=U(\vec{\alpha}+\vec{\xi}) .
$$

It is very important at this point to note that the lensing effect does not produce any polarization nor rotate the Stokes parameter. In this regime its effect reduces to a simple deformation of the polarization patterns, similar to the temperature maps. This is the mechanism by which the geometrical properties of the polarization field are changed.

To see that we have to consider the electric $(E)$ and magnetic $(B)$ components instead of the Stokes parameters. The $E$ component is the scalar part (similar to the convergence field) and $B$ is the pseudo scalar part. This decomposition is common place in CMB physics because the scalar perturbations, which give the dominant contributions to the temperature anisotropies, cannot induce $B$ type polarization. Only tensor modes (e.g. gravity waves) can do it. In inflationary mechanisms one expects those modes to be present but subdominant, specially at small scales.

In the small angle limit, when the perturbations can be approximated as plane waves, we can write a perturbation description of the lensing effect on electric and magnetic components of the polarization. At leading order one obtains (Benabed et al. 2001):

$$
\begin{aligned}
& \Delta \hat{E}=\Delta E+\xi^{i} \partial_{i} \Delta E-2 \kappa \Delta E-2 \delta_{i j}^{\mathrm{K}}\left(\gamma^{i} \Delta P^{j}+\gamma_{, k}^{i} P^{j, k}\right)+O\left(\gamma^{2}\right) \\
& \Delta \hat{B}=\Delta B+\xi^{i} \partial_{i} \Delta B-2 \kappa \Delta B-2 \epsilon_{i j}\left(\gamma^{i} \Delta P^{j}+\gamma_{, k}^{i} P^{j, k}\right)+O\left(\gamma^{2}\right),
\end{aligned}
$$

where $P^{i}$ are the components of the polarization field, $\gamma$ and $\kappa$ are respectively the shear and convergence field, delta $a_{i j}^{\mathrm{K}}$ is the Kronecker symbol and $\epsilon_{i j}$ is the totally antisymmetric tensor. As a result one expects the $E$ power spectrum to be transformed in a manner roughly similar to that of the temperature field. More importantly it also implies that $B$ modes can be created out of $E$ modes (Zaldarriaga and Seljak 1998, Benabed \& Bernardeau 2000, Benabed et al. 2001).

The consequence is that there is now a significant $B$ mode power spectrum even if it was not present in the primordial polarization field. The phenomenological consequences are depicted on Fig. 4 which shows the induced $B$ power spectrum compared to one that would be expected in inflation (note that the relative amplitude of the primordial $B$ modes to the anisotropies is model dependent. It depends in particular on the slow-roll parameters of the inflationary scenario. This is not the case for the amplitude of the 


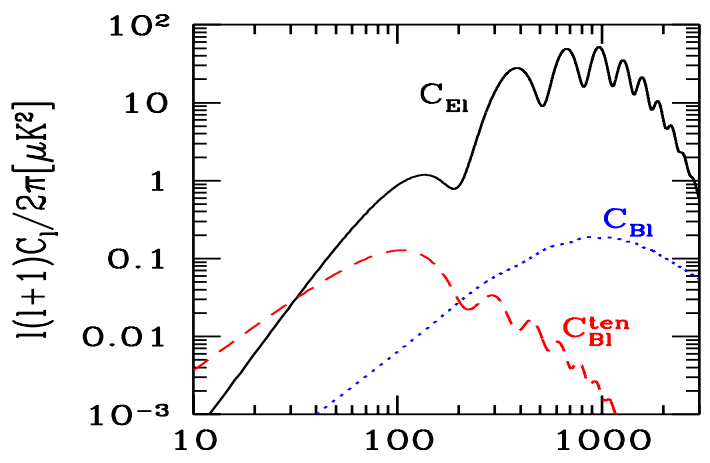

Figure 4. The B-mode power spectrum (dotted line) as induced by the lenses (from Zaldarriaga and Seljak 1998).

$E$ modes that are essentially determined by the scattering processes at recombination time.)

\section{Mode coupling effects in temperature maps}

Compared to detections on background galaxies, the analysis of the lens effects on temperature filed requires sophisticated tools because the number of background structure is much smaller though they have much better defined statistical properties. The primordial temperature patches on the CMB sky are indeed known only statistically and have a large angular correlation length. In which way, then, can the lens effects be revealed? Actually lensed CMB maps can be seen as collections of temperature patches of different sizes and shapes which, or only a fraction of which, are displaced or deformed. Although this is slightly arbitrary, two effects can be distinguished in the way sizes and shapes of patches are affected,

- the shear effect that deforms, stretches out temperature patches in the shear direction,

- the magnification effect that globally enlarges or shrinks those patches.

The local deformations of the temperature patches are however a priori difficult to disentangle from the actual primordial intrinsic temperature fluctuations. What will make then the effects detectable is the fact that close patches will be deformed in a similar way (when they are seen through a unique lens), and the excess of these close rare features cannot be accounted from a Gaussian field. It is thus possible to quantify their presence by statistical indicators. The power spectrum is of course not adapted to take into account the apparition of such non-Gaussian features. For that matter the high-order correlation functions, that are all identically zero for pure Gaussian fields, are extremely precious. Indeed these higher-order correlation functions contain information about shapes, and their derivations can be pursued completely with Perturbation Theory techniques. In the following the focus of the analysis will be on the first non vanishing correlation function, the four-point one.

It is quite easy to see that the weak-lensing effects do not introduce a three-point correlation function. The lens effect is indeed symmetric in terms of positive or negative temperature fluctuations $\dagger$.

$\dagger$ As mentioned in the following a non-zero three point function can however be induced through other secondary effects such as the integrated Sachs-Wolfe effects or Sunyaev-Seldovich effects. 
The first non trivial high order correlation function is thus the four-point correlation function. At this stage it is important to have in mind that the observable quantity is the connected part, $\left\langle T\left(\vec{\gamma}_{1}\right) T\left(\vec{\gamma}_{2}\right) T\left(\vec{\gamma}_{3}\right) T\left(\vec{\gamma}_{4}\right)\right\rangle_{c}$, of the ensemble average, $\left\langle T\left(\vec{\gamma}_{1}\right) T\left(\vec{\gamma}_{2}\right) T\left(\vec{\gamma}_{3}\right)\right.$ $\left.T\left(\vec{\gamma}_{4}\right)\right\rangle$, that is the part which is obtained when the products of two point correlation functions that can be built have been subtracted out,

$$
\begin{array}{r}
\left\langle T\left(\vec{\gamma}_{1}\right) T\left(\vec{\gamma}_{2}\right) T\left(\vec{\gamma}_{3}\right) T\left(\vec{\gamma}_{4}\right)\right\rangle_{c} \equiv\left\langle T\left(\vec{\gamma}_{1}\right) T\left(\vec{\gamma}_{2}\right) T\left(\vec{\gamma}_{3}\right) T\left(\vec{\gamma}_{4}\right)\right\rangle- \\
{\left[\left\langle T\left(\vec{\gamma}_{1}\right) T\left(\vec{\gamma}_{2}\right)\right\rangle\left\langle T\left(\vec{\gamma}_{3}\right) T\left(\vec{\gamma}_{4}\right)\right\rangle+\text { perm. (2 other terms) }\right] .}
\end{array}
$$

The connected part is obviously zero for the primordial field: it is a direct consequence of its Gaussian nature. The dominant term, in terms of weak lensing effects, is thus given by,

$$
\begin{aligned}
\left\langle T\left(\vec{\gamma}_{1}\right) T\left(\vec{\gamma}_{2}\right) T\left(\vec{\gamma}_{3}\right) T\left(\vec{\gamma}_{4}\right)\right\rangle_{c} & \equiv\left\langle T\left(\vec{\gamma}_{1}\right) T_{, i}\left(\vec{\gamma}_{2}\right)\right\rangle\left\langle\xi_{i}\left(\vec{\gamma}_{2}\right) \xi_{j}\left(\vec{\gamma}_{3}\right)\right\rangle\left\langle T_{, j}\left(\vec{\gamma}_{3}\right) T\left(\vec{\gamma}_{4}\right)\right\rangle \\
& + \text { perm. (11 other terms) }
\end{aligned}
$$

Roughly speaking it means that the four-point correlation function, in units of the square of the second, is proportional to the weak lensing angular correlation function.

The effects of lenses can be much more easily handled in Fourier space. The existence of a non-vanishing four point function in real space means that there is a tri-spectrum for the temperature field $T\left(\mathbf{l}_{1}, \mathbf{l}_{2}, \mathbf{l}_{3}, \mathbf{l}_{4}\right)$ defined as,

$$
\left\langle T\left(\mathbf{l}_{1}\right) T\left(\mathbf{l}_{2}\right) T\left(\mathbf{l}_{3}\right) T\left(\mathbf{l}_{4}\right)\right\rangle=\delta_{\text {Dirac }}\left(\mathbf{l}_{1}+\mathbf{l}_{2}+\mathbf{l}_{3}+\mathbf{l}_{4}\right) T\left(\mathbf{l}_{1}, \mathbf{l}_{2}, \mathbf{l}_{3}, \mathbf{l}_{4}\right)
$$

The expression of the tri-spectrum can be easily obtained at leading order in the convergence field from the relation (2.3). It reads,

$$
T\left(\mathbf{l}_{1}, \mathbf{l}_{2}, \mathbf{l}_{3}, \mathbf{l}_{4}\right)=\frac{\left(\mathbf{l}_{1} \cdot \mathbf{l}_{3}\right)\left(\mathbf{l}_{2} \cdot \mathbf{l}_{3}\right)}{l_{3}^{2}} C^{T}\left(l_{1}\right) C^{T}\left(l_{2}\right) P_{\kappa}\left(l_{3}\right)+\text { sym. }
$$

It exhibits a specific geometrical dependence which is obviously expected to be visible at the level of the four-point function of the temperature field. The latter can be expressed in terms of the angular derivative of the two-point temperature correlation function which reads,

$$
\frac{\mathrm{d}}{\mathrm{d} \theta} C^{T}(\theta)=-\int \frac{l^{2} \mathrm{~d} l}{2 \pi} C^{T}(l) J_{1}(l \theta)
$$

and with the angular correlation of the displacement field $C_{d}(\theta)$,

$$
C_{d}(\theta)=\int \frac{l \mathrm{~d} l}{2 \pi} J_{0}(l \theta) P_{\kappa}(l)
$$

and with the related quantity,

$$
\tilde{C}_{d}(\theta)=\int \frac{l \mathrm{~d} l}{2 \pi} J_{2}(l \theta) P_{\kappa}(l) .
$$

It leads to,

$$
\begin{gathered}
\left\langle T\left(\vec{\gamma}_{1}\right) T\left(\vec{\gamma}_{2}\right) T\left(\vec{\gamma}_{3}\right) T\left(\vec{\gamma}_{4}\right)\right\rangle_{c}=\frac{1}{2} \frac{\mathrm{d}}{\mathrm{d} \theta} C^{T}\left(\gamma_{12}\right) \frac{\mathrm{d}}{\mathrm{d} \theta} C^{T}\left(\gamma_{34}\right) \times \\
{\left[C_{d}\left(\gamma_{23}\right) \cos \left(\varphi_{12}-\varphi_{34}\right)-\tilde{C}_{d}\left(\gamma_{23}\right) \cos \left(\varphi_{12}+\varphi_{34}\right)\right]+\text { perm. (11 other terms), }}
\end{gathered}
$$

where $\varphi_{12}$ is the angle between $\vec{\gamma}_{12}$ and $\vec{\gamma}_{23}$ and $\varphi_{34}$ is the angle between $\vec{\gamma}_{43}$ and $\vec{\gamma}_{32}$ (see Fig. 1). Two terms are thus involved. The a priori dominant term is the one in $D_{0}$, 


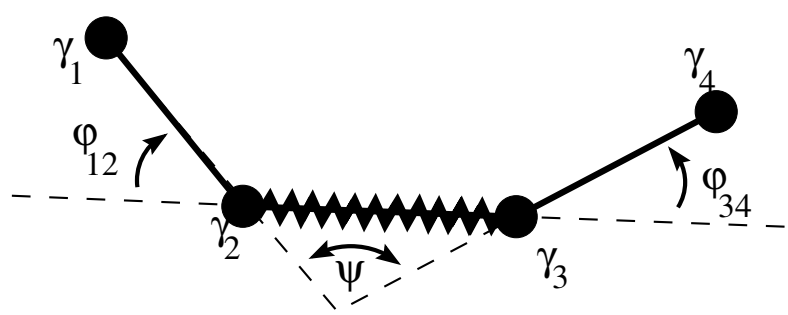

Figure 5. Description of the angles intervening in the expression (3.8) of the four-point correlation function. The thick solid line materializes the $\mathrm{d} C^{T}(\theta) / \mathrm{d} \theta$ factor, whereas the hatched lines represents the correlation functions of the displacement field.

and it is weighted by the cosine of the angle $\varphi_{12}-\varphi_{34} \equiv \psi$ (see Fig. 1), that is the angle between the directions $\vec{\gamma}_{12}$ and $\vec{\gamma}_{34}$ on the sky. It gives a clear geometrical dependence for the four point-correlation function. However, one should have in mind that 11 other terms have to be taken into account in this calculation. This signal may therefore be masked by other geometric dependencies.

Quantitative calculations can be done for specific cosmological models (see next subsection). However, one obvious problem for a practical determination of this correlation function is that it depends on 5 different variables. It is therefore interesting to reduce the number of variables while by considering simplified geometries that still preserve the geometrical signature of the lens effects.

\subsection{A peculiar case: when two directions coincide}

One example is when two points coincide, that is the expression of $\left\langle T\left(\vec{\gamma}_{1}\right)\left[T\left(\vec{\gamma}_{2}\right)\right]^{2} T\left(\vec{\gamma}_{3}\right)\right\rangle_{c}$. This notation is actually a bit oversimplified since the local temperature fluctuations are filtered by the used apparatus. One should thus have in mind that the two directions denoted $\vec{\gamma}_{2}$ are actually close random directions in a beam centered on $\vec{\gamma}_{2}$.

Of course, once again, many terms are contributing to this ensemble average but let us first concentrate on the case where the connection between the two $T\left(\vec{\gamma}_{2}\right)$ is made by the lens coupling term. In such a case one can see that $\psi$ is given by the angle between $\vec{\gamma}_{2}-\vec{\gamma}_{1}$ and $\vec{\gamma}_{3}-\vec{\gamma}_{2}$ and is not affected by the smoothing. This is not the case for the term in $\cos \left(\varphi_{12}+\varphi_{34}\right)$ which is expected to almost vanish because it is averaged to zero and is indeed found to give a negligible contribution. This peculiar correlation function is therefore proportional to the cosine of the angle, and to the autocorrelation function of the displacement field.

It is then interesting to define the function,

$$
\kappa_{4}\left(\vec{\gamma}_{12}, \vec{\gamma}_{23}\right)=\frac{\left\langle T\left(\vec{\gamma}_{1}\right)\left[T\left(\vec{\gamma}_{2}\right)\right]^{2} T\left(\vec{\gamma}_{3}\right)\right\rangle_{c}}{\left\langle T\left(\vec{\gamma}_{1}\right) T\left(\vec{\gamma}_{2}\right)\right\rangle\left\langle T\left(\vec{\gamma}_{2}\right) T\left(\vec{\gamma}_{3}\right)\right\rangle},
$$

which is a dimensionless quantity. It is directly proportional to the large-scale structure power spectrum, with a known dependence on the shape of the anisotropy power spectrum. This quantity would thus be a measure the weak lensing effects in CMB maps. According to the previous considerations, the leading contribution of this term is,

$$
\begin{gathered}
\kappa_{4}\left(\vec{\gamma}_{12}, \vec{\gamma}_{23}\right)=\frac{\mathrm{d}}{\mathrm{d} \theta} \log \left[C\left(\gamma_{12}\right)\right] \frac{\mathrm{d}}{\mathrm{d} \theta} \log \left[C\left(\gamma_{23}\right)\right] \cos (\psi) \times \\
{\left[\bar{D}_{0}\left(\theta_{0}\right)+D_{0}\left(\gamma_{13}\right)-D_{0}\left(\gamma_{12}\right)-D_{0}\left(\gamma_{23}\right)\right] .}
\end{gathered}
$$




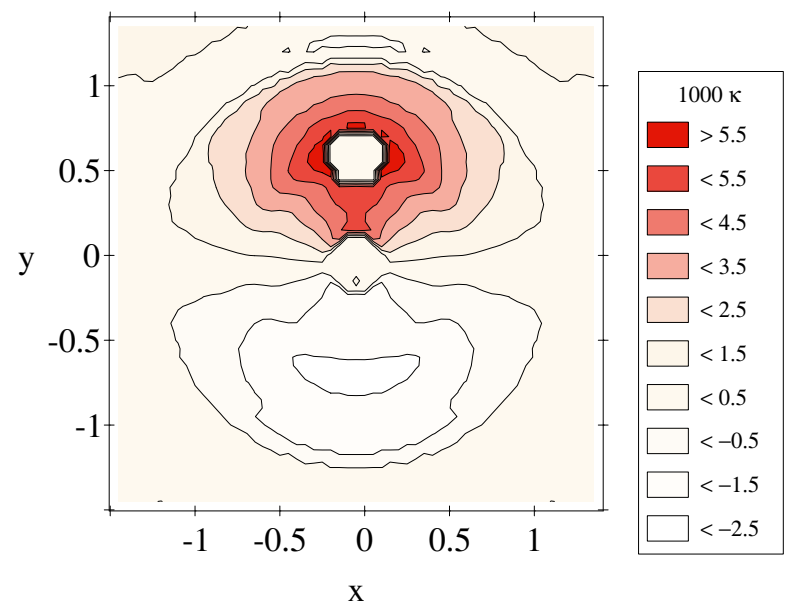

Figure 6. Contour plot of the function $\kappa_{4}\left(\vec{\gamma}_{12}, \vec{\gamma}_{23}\right)$ (eq. 3.9) as a function of the relative position (in degrees) of $\vec{\gamma}_{3}$ when $\vec{\gamma}_{2}$ is the central point of the graph and $\vec{\gamma}_{1}$ is at the coordinates $x=0$, $y=+0.63 \mathrm{deg}$. The value of $\kappa_{4}$ has been multiplied by 1000 .

where $\bar{D}_{0}$ is the averaged displacement field over the scale $\theta_{0}$. A $2 \mathrm{D}$ contour plot of the function $\kappa_{4}\left(\vec{\gamma}_{12}, \vec{\gamma}_{23}\right)$ is presented on Fig. 6 for a peculiar cosmological case (standard CDM).

If the four-point function is the most immediate signature of the lens effects, clearly as soon as the temperature field is not Gaussian, many other signatures of these nonGaussian properties can be considered. In the literature, people have examined the hot (or cold) spot correlation function (Takada et al. 2000), the ellipticity statistics (Bernardeau (1998)), the genus and Minkowski functional (Schmalzing et al. 2000, Takada 2001) the cumulant properties of temperature probability distribution functions (Kesden et al. 2002). None of these signatures however can provide for a large signal to noise detection (even for a Planck size experiment). The reasons is that the number of structures available on the CMB sky is intrinsically limited.

What the result on the four point function shows however is that in principle one should have access to the convergence power spectrum. What it does not, is to take into account the full richness of the CMB sky, e.g. its polarization patterns.

\section{Reconstruction methods from temperature and polarization maps}

In spirit the development of methods for the reconstruction of mass maps from CMB observations is based on the ideas that the CMB patterns can be averaged out to reveal the lensing signal. In some sense what is looked for is an optimal way of stacking the four-point functions to reveal the lensing signal. The method is finally not so different from what is done for galaxy shear measurements; the difference being that one cannot rely here on a large number of background sources but on subtle changes in the shape correlations.

Two criteria should used to gauge the efficiency of such methods: their ability to provide us with unbiased estimators of the convergence field and how the signal compares to the "CMB noise" level. The latter is obviously dependent on the number of observed structures which is directly related to the angular resolution of the surveys. 


$$
\begin{array}{ll}
x x^{\prime} & K_{x x^{\prime}}\left(\mathbf{l}_{1}, \mathbf{l}_{2}\right) \\
\hline \Theta \Theta & \tilde{C}_{l_{1}}^{\Theta \Theta}\left(\mathbf{L} \cdot \mathbf{l}_{1}\right)+\tilde{C}_{l_{2}}^{\Theta \Theta}\left(\mathbf{L} \cdot \mathbf{l}_{2}\right) \\
\Theta E & \tilde{C}_{l_{1}}^{\Theta E} \cos \varphi_{\mathbf{l}_{1} \mathbf{l}_{2}}\left(\mathbf{L} \cdot \mathbf{l}_{1}\right)+\tilde{C}_{l_{2}}^{\Theta E}\left(\mathbf{L} \cdot \mathbf{l}_{2}\right) \\
\Theta B & \tilde{C}_{l_{1}}^{\Theta E} \sin 2 \varphi_{\mathbf{l}_{1} \mathbf{l}_{2}}\left(\mathbf{L} \cdot \mathbf{l}_{1}\right) \\
E E & {\left[\tilde{C}_{l_{1}}^{E E}\left(\mathbf{L} \cdot \mathbf{l}_{1}\right)+\tilde{C}_{l_{2}}^{E E}\left(\mathbf{L} \cdot \mathbf{l}_{2}\right)\right] \cos 2 \varphi_{\mathbf{l}_{1} \mathbf{l}_{2}}} \\
E B & {\left[\tilde{C}_{l_{1}}^{E E}\left(\mathbf{L} \cdot \mathbf{l}_{1}\right)-\tilde{C}_{l_{2}}^{B B}\left(\mathbf{L} \cdot \mathbf{l}_{2}\right)\right] \sin 2 \varphi_{\mathbf{l}_{1} \mathbf{l}_{2}}} \\
B B & {\left[\tilde{C}_{l_{1}}^{B B}\left(\mathbf{L} \cdot \mathbf{l}_{1}\right)+\tilde{C}_{l_{2}}^{B B}\left(\mathbf{L} \cdot \mathbf{l}_{2}\right)\right] \cos 2 \varphi_{\mathbf{l}_{1} \mathbf{l}_{2}}}
\end{array}
$$

Table 1. Expression of the function $K_{x x^{\prime}}$ appearing in Eq. (4.1) as a function of the field nature. $\tilde{C}_{l}^{x x^{\prime}}$ is the power $x x^{\prime}$-spectrum of the unlensed fields.

The most simple estimators are based on quadratic quantities. This reflects the fact that the first non vanishing high order correlation functions is the fourth point one. A general framework for building such estimators has been presented by $\mathrm{Hu} \&$ Okamoto (2002).

\subsection{Minimum Variance Estimators}

As it clearly appears in Eq. (2.3), lensing effects mix and therefore correlate Fourier modes across a range defined by the strength in the deflection field $\phi(\mathrm{Hu} 2000)$. If it were possible to average over an ensemble of realizations of the temperature and polarization fields but with a fixed lensing field one would obtain correlation functions of the form,

$$
\left\langle x(\mathbf{l}) x^{\prime}\left(\mathbf{l}^{\prime}\right)\right\rangle_{\mathrm{CMB}}=K_{x x^{\prime}}\left(\mathbf{l}, \mathbf{l}^{\prime}\right) \phi(\mathbf{L}),
$$

where $x$ and $x^{\prime}$ are either $T, E$ or $B$ fields, $\mathbf{L}=\mathbf{l}+\mathbf{l}^{\prime}$ and $K_{x x^{\prime}}\left(\mathbf{l}, \mathbf{l}^{\prime}\right)$ is a kernel function that encodes the lensing effects depending on the fields. The expressions of the functions $K_{x x^{\prime}}$ is recalled in table 1 . Clearly if such averages were possible they would provide us with estimates of the deflecting potential.

However the two-point correlations of the CMB Fourier modes themselves cannot be used to reconstruct the deflection potential: $\phi$ being also statistically isotropic a complete ensemble average $\langle\phi(L)\rangle$ obviously vanishes. The Eq. (4.1) does suggest however that an appropriate average over pairs of multipole moments can be used to estimate the Fourier modes of the deflection field $d(\mathbf{L})$.

As shown in $\mathrm{Hu} \&$ Okamoto (2002) this can be achieved by defining a proper weighting of the moments that takes the form

$$
d_{x x^{\prime}}(\mathbf{L})=\frac{A_{x x^{\prime}}(L)}{L} \int \frac{\mathrm{d}^{2} \mathbf{l}_{1}}{(2 \pi)^{2}} x\left(\mathbf{l}_{1}\right) x^{\prime}\left(\mathbf{l}_{2}\right) F_{x x^{\prime}}\left(\mathbf{l}_{1}, \mathbf{l}_{2}\right),
$$

where $\mathbf{l}_{2}=\mathbf{l}-\mathbf{l}_{1}$ and the normalization factor $A_{x x^{\prime}}$ is chosen so that

$$
\left\langle d_{\alpha}(\mathbf{L})\right\rangle_{\mathrm{CMB}}=d(\mathbf{L}) \equiv L \phi(\mathbf{L}) .
$$

Each combination for $x$ and $x^{\prime}$ provides with an estimate of $d(\mathbf{L})$. Each of which ought to be the most likely deflection mode that can be inferred from the $x$ or $x^{\prime}$ measurements. The whole game is then to choose the filter $F$ in a way that optimizes the signal that is that minimizes the intrinsic variance $\left\langle d_{x x^{\prime}}^{*}(L) d_{x x^{\prime}}(L)\right\rangle$ of the estimated deflection mode, subject to the normalization constraint. This is a mathematically well defined problem 


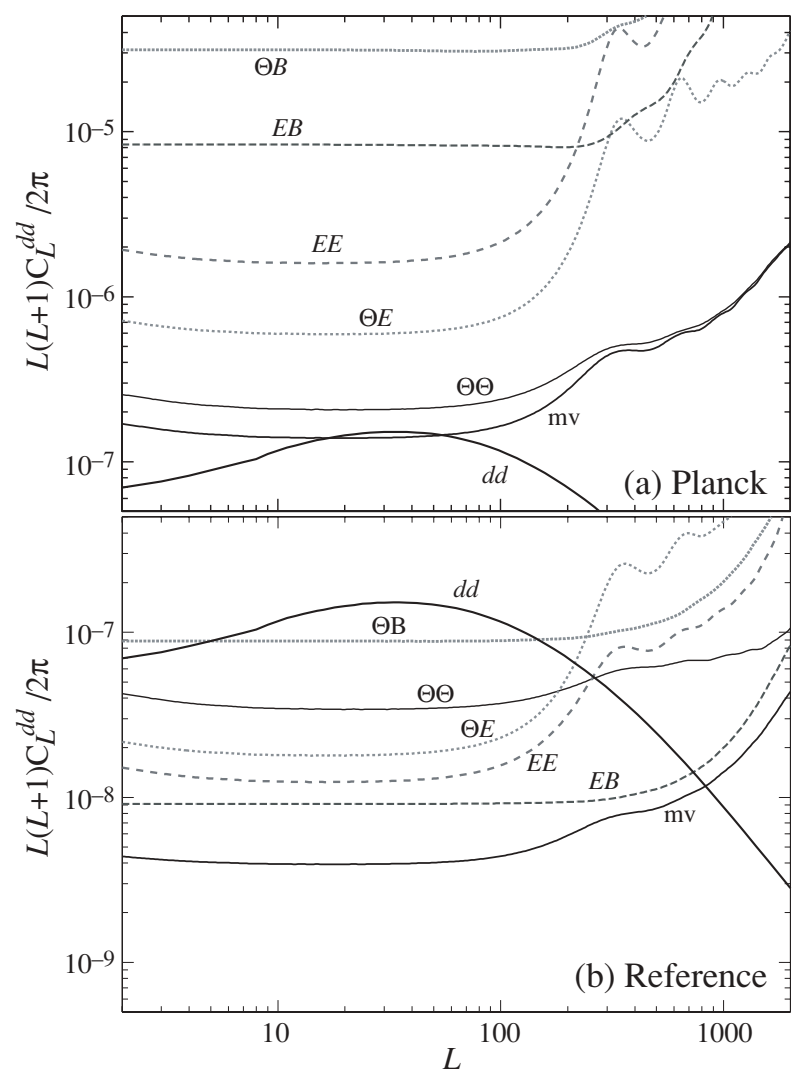

Figure 7. Deflection signal $(d d)$ and noise power spectra of the quadratic estimators and their minimum variance (mv) combination: (a) Planck experiment (b) reference experiment. As the sensitivity of the experiment improves the best quadratic estimator switches from $\Theta \Theta$ to $E B$. Only the $E B$-estimator can reconstruct the mass distribution at $L \gtrsim 200$.

the solution of which reads,

$$
F_{x x^{\prime}}\left(\mathbf{l}_{1}, \mathbf{l}_{2}\right)=\frac{C_{l_{1}}^{x^{\prime} x^{\prime}} C_{l_{2}}^{x x} K_{x x^{\prime}}\left(\mathbf{l}_{1}, \mathbf{l}_{2}\right)-C_{l_{1}}^{x x^{\prime}} C_{l_{2}}^{x x^{\prime}} K_{x x^{\prime}}\left(\mathbf{l}_{2}, \mathbf{l}_{1}\right)}{C_{l_{1}}^{x x} C_{l_{2}}^{x^{\prime} x^{\prime}} C_{l_{1}}^{x^{\prime} x^{\prime}} C_{l_{2}}^{x x}-\left(C_{l_{1}}^{x x^{\prime}} C_{l_{2}}^{x x^{\prime}}\right)^{2}} .
$$

The expected signal is then to be compared to the noise properties of these estimators. They follow from the expression of the deflection correlators that take into account both the cosmic variance of the fields and the noise variance of the experiment,

$$
\left\langle d_{x x^{\prime}}^{*}(\mathbf{L}) d_{y y^{\prime}}\left(\mathbf{L}^{\prime}\right)\right\rangle=(2 \pi)^{2} \delta\left(\mathbf{L}-\mathbf{L}^{\prime}\right)\left[C_{L}^{d d}+N_{\left(x x^{\prime}\right)\left(y y^{\prime}\right)}(L)\right],
$$

where

$$
\begin{aligned}
N_{\left(x x^{\prime}\right)\left(y y^{\prime}\right)}(L)= & \frac{1}{L^{2}} A_{x x^{\prime}}(L) A_{y y^{\prime}}(L) \int \frac{\mathrm{d}^{2} \mathbf{l}_{1}}{(2 \pi)^{2}} F_{x x^{\prime}}\left(\mathbf{l}_{1}, \mathbf{l}_{2}\right) \\
& \times\left(F_{y y^{\prime}}\left(\mathbf{l}_{1}, \mathbf{l}_{2}\right) C_{l_{1}}^{x y} C_{l_{2}}^{x^{\prime} y^{\prime}}+F_{y y^{\prime}}\left(\mathbf{l}_{2}, \mathbf{l}_{1}\right) C_{l_{1}}^{x y^{\prime}} C_{l_{2}}^{x^{\prime} y}\right) .
\end{aligned}
$$

Notice that for the minimum variance filter

$$
N_{\alpha \alpha}(L)=A_{\alpha}(L) .
$$

In Fig. 7, we compare the signal and noise power spectra for the Planck experiment and 


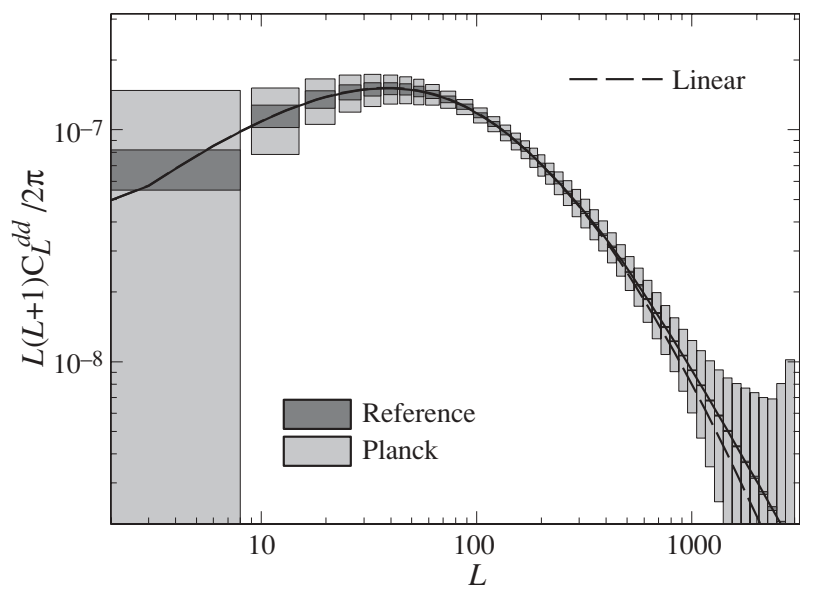

Figure 8. Statistical errors achievable on the deflection power spectrum with the Planck $\left(f_{\text {sky }}=0.65\right)$ and reference experiments $\left(f_{\text {sky }}=1\right)$. Boxes represent band averaging width and $1 \sigma$ errors. The polarization information in the reference experiment allows for a cosmic variance limited measurement of the projected power spectrum out to $L \sim 1000$. In this regime, the fluctuations are almost completely linear (dashed lines), from $\mathrm{Hu} \&$ Okamoto (2002).

the reference experiment. The latter is such that $\Delta_{T}=\Delta_{P} / \sqrt{2}=1 \mu \mathrm{K}$-arcmin and with an angular resolution of $4^{\prime}$.

The reconstruction that can potentially be achieved are presented on Fig. 8 for Planck and for the reference experiment.

\subsection{Maximum likelihood methods}

Although efficient the previous method has its own intrinsic limitation. In particular it does not take into account the higher order couplings that the lens effects induce. If it is not a critical issue at angular scales of interest (say $l \leqslant 3500$ ) for the temperature field, the issue is more subtle for the polarization field. In particular the argument for optimality of the quadratic estimator presented by Hirata \& Seljak (2003a) does not apply to polarization since the $B$-mode power is dramatically increased by lensing. The aim of this section is to underline that likelihood-based estimators for lensing using the CMB polarization can significantly improve on the quadratic estimator (Hirata \& Seljak 2003b). Indeed, as noise is decreased the accuracy of CMB lensing reconstruction continues to improve without bound. Conceptually this is because if the lensed polarization is measured with zero noise, then the equation $B_{\text {unlensed }}=0$ can be solved (except possibly for some degenerate modes, Benabed et al. 2001) for the projected matter density with zero noise. The equation $B_{\text {unlensed }}=0$ is however ill-behaved in the presence of instrument noise and cannot be inverted; fortunately, the likelihood formalism easily incorporates noise and in practice regularizes the problem.

It goes beyond the scope of these notes to present in details the formalism of such a method. The performance of this method is however illustrated on Fig. 9. It shows that the small scale modes can be recovered much more accurately with maximum likelihood methods. 


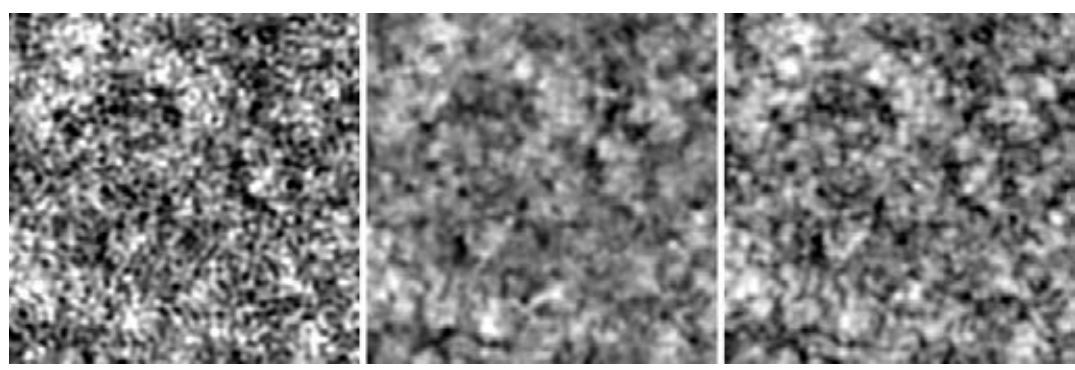

Figure 9. A simulated reconstruction of the lensing convergence using polarization and a reference experiment $\left(\mu \mathrm{K} \operatorname{arcmin}=1.41, \theta_{F W H M}=4^{\prime}, l_{\max }=2020\right)$. In the left panel, we display the realization of the convergence field $\kappa$ used to produce the simulated CMB. The reconstructions using the Wiener-filtered quadratic estimator and the iterative estimator are shown in the center and right panels, respectively. These frames are each $8^{\circ} 32^{\prime}$ in angular width (From Hirata \& Seljak 2003b).

\section{Lens induced CMB-large scale structure correlations}

\subsection{Motivations}

Even with the most precise experiments it is clear that clean detections of $B$ component will be difficult to obtain. The magnetic polarization amplitude induced with such a mechanism is expected to be one order of magnitude below the electric one. Besides even if we knew that there is a window in angular scale where the other secondary effects will not interfere too much with the detection of the lens-induced $B$, few is still known about removing the foregrounds to obtain clean maps reconstruction algorithms would require.

These considerations call for complementary data sets to compare $B$ and generally CMB lenses with. Although the source plane for galaxy lensing surveys is much closer than for the lensed CMB fluctuations, we expect to have a significant overlapping region in the two redshift lens distributions, so that weak lensing surveys can map a fair fraction of the line-of-sight CMB lenses. Consequently, weak lensing surveys can potentially provide us with shear maps correlated with for instance CMB $B$ maps, but with have different geometrical degeneracy, noise sources and systematics.

The correlation strength between the lensing effects at two different redshifts can easily be evaluated. We define $r$ as the cross-correlation coefficient between two lens planes:

$$
r\left(z_{\mathrm{GAL}}\right)=\frac{\left\langle\kappa \kappa_{\mathrm{GAL}}\right\rangle}{\sqrt{\left\langle\kappa^{2}\right\rangle\left\langle\kappa_{\mathrm{GAL}}^{2}\right\rangle}} .
$$

In a broad range of realistic cases (see tab. 2), $r \sim 40 \%$. To take advantage of this large overlapping we will consider quantity that cross correlates the CMB $B$ field and galaxy surveys. Moreover, cross-correlation observations are expected to be insensitive to noises in weak lensing surveys and in CMB polarization maps. This idea can be explored for temperature maps (in van Waerbeke et al. 2000) as well as for polarization maps (in Benabed et al. 2001) taking advantage of the specific geometrical dependences uncovered in the previous section.

\subsection{From the temperature field alone}

Such correlations have to rely on subtle differences in the shape patterns of the CMB temperature maps. More specifically Bernardeau (1998) investigated the effect of lensing by the large-scale structures on the distribution of the CMB ellipticities. The consequences are the same as for the lensed distant galaxies: the gravitational distortion induces an excess of elongated structures of CMB ellipticities. The intrinsic CMB ellipticity 


\begin{tabular}{ccc}
$r$ coefficient & $z_{\mathrm{GAL}}=1$ & $z_{\mathrm{GAL}}=2$ \\
\hline EdS, Linear & 0.42 & 0.60 \\
$\Omega=0.3, \Lambda=0.7$, Linear & 0.31 & 0.50 \\
$\Omega=0.3, \Lambda=0.7$, Non Linear & 0.40 & 0.59
\end{tabular}

Table 2. values of $r$, the cross-correlation between two source planes $\left(z_{\mathrm{GAL}}\right.$ and $\left.z_{\mathrm{CMB}}=1100\right)$ for different models. For the non-linear scale the adopted filter scale is 2 arcmin for both weak lensing survey and Cosmic Microwave Background observations and the non-linear $P(k)$ has been computed using Peacock and Dodds method (Peaock \& Dodds 1996).

distribution being known for a Gaussian field (e.g. Bond \& Efstathiou 1987) it is then possible to compute the lensed distribution. The lensed distribution is unfortunately rather close to the unlensed one, in particular because the smoothing caused by the CMB beam tends to circularizes the local structures. The orientation of the local ellipticity is however expected to be much more robust against the smoothing effects and therefore more efficient in tracing the lens effects. Due to the low number density of structures on CMB maps such effects can be hardly detected in CMB maps alone, so in this section the possible cross-correlation of CMB ellipticities with the distant galaxy ellipticities is reviewed.

This paragraph is devoted to the description of the expected correlation between the CMB ellipticities and those of distant galaxies. We indeed expect their relative angle to be not-uniformly distributed unlike what would happen if there were no lensing effects. We examine here the amplitude and the observability of this effect.

At any position on the CMB temperature map, we can define an ellipticity e from the curvature of the temperature field $\delta_{T}$ :

$$
\mathbf{e}=\left(\frac{\partial_{x}^{2} \delta_{T}-\partial_{y}^{2} \delta_{T}}{\partial_{x}^{2} \delta_{T}+\partial_{y}^{2} \delta_{T}} ; \frac{2 \partial_{x y} \delta_{T}}{\partial_{x}^{2} \delta_{T}+\partial_{y}^{2} \delta_{T}}\right) .
$$

This relation is similar to the ellipticity of a galaxy defined from its second order moments. A peak of temperature with the same curvature on both axis has a zero ellipticity, but in opposition with the galaxies, the CMB ellipticity can take any value between zero (circular peaks) and infinity (symmetric saddle points). However, it is always meaningful to define the orientation of the CMB ellipticity $\theta_{\mathbf{e}}=\arctan \left(e_{2} / e_{1}\right)$, which runs from 0 to $2 \pi$. The gravitational lensing effect tends to stretch the structures and therefore to produce an excess of elongated structures relative to the number of rather round objects as shown in Bernardeau (1998). The lenses tend also to align the CMB ellipticity with the shear $\vec{\gamma}(\theta)$ acting on the CMB at the angular position $\theta$. This is similar to the effect which occurs on the ellipticity of distant galaxies, although the corresponding shear $\vec{\gamma}_{\mathrm{GAL}}(\theta)$ cannot be identified with $\vec{\gamma}(\theta)$ since the galaxies are at much lower redshift. In the following, we will label local lensing quantities, such as what one can obtain from lensing reconstruction on galaxy surveys, with a GAL index. The variables $\vec{\gamma}(\theta)$ and $\vec{\gamma}_{\mathrm{GAL}}(\theta)$ are correlated because the light coming from either the CMB or the distant galaxies are passing through the same portion of low-redshift Universe, and consequently, for a given line-of-sight $\theta$, the CMB ellipticities are preferentially aligned with the distant galaxies.

The relative angle between the two direction was found to be given by

$$
\mathcal{P}\left(\theta_{\mathrm{GAL}}\right) \mathrm{d} \theta_{\mathrm{GAL}}=\frac{\mathrm{d} \theta_{\mathrm{GAL}}}{2 \pi}\left(1+3 \sqrt{\frac{\pi}{2}} \frac{\left\langle\kappa \kappa_{\mathrm{GAL}}\right\rangle}{\left\langle\kappa_{\mathrm{GAL}}^{2}\right\rangle} \cos \left(\theta_{\mathrm{GAL}}\right)\right) .
$$

Fig. 10 shows the amplitude of this effect for different cosmological models and different smoothing scales. We assumed a CDM power spectrum taking into account its non-linear 


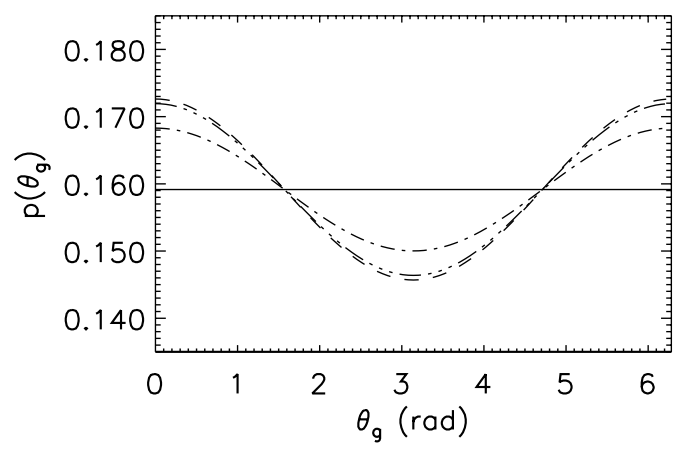

Figure 10. Probability distribution function of the relative orientation $\theta_{\mathrm{GAL}}$ between the CMB ellipticity and the sheared distant galaxies (Eq.5.3). The beam size as well as the smoothing length of the convergence $\kappa_{\mathrm{GAL}}$ are $\theta_{0}^{\mathrm{CMB}}=10^{\prime}$. The horizontal solid line represents the uniform distribution of $\theta_{\mathrm{GAL}}$ in the un-lensed case. The dot-dashed line is for $\Omega_{0}=0.3, \Omega_{\Lambda}=0, \sigma_{8}=1$, the triple dot-dashed line for $\Omega_{0}=0.3, \Omega_{\Lambda}=0.7, \sigma_{8}=1$ and the dashed line for $\Omega_{0}=1$, $\Omega_{\Lambda}=0, \sigma_{8}=0.6$ (from van Waerbeke et al. 2000).

\begin{tabular}{lccc} 
Model & $\left\langle\cos \left(\theta_{\mathrm{GAL}}\right)\right\rangle$ & $S / N$ for $\theta_{0}=5^{\prime}$ & $S / N$ for $\theta_{0}=10^{\prime}$ \\
\hline standard-CDM $\left(900 \mathrm{deg}^{2}\right)$ & $0.057\left(\theta_{0}=5^{\prime}\right)$ & 5.4 & 3.3 \\
$\Lambda$-CDM (900 $\left.\mathrm{deg}^{2}\right)$ & $0.054\left(\theta_{0}=5^{\prime}\right)$ & 6.7 & 3.9 \\
Open-CDM $\left(900 \mathrm{deg}^{2}\right)$ & $0.040\left(\theta_{0}=5^{\prime}\right)$ & 4.4 & 2.3
\end{tabular}

Table 3. Values of $\left\langle\cos \left(\theta_{\mathrm{GAL}}\right)\right\rangle$ and its signal-to-noise estimation.

evolution (using the formula given in Peaock \& Dodds 1996). The deviation from a uniform distribution can be as large as $10 \%$, and the effect seems mostly sensitive to the curvature of the Universe rather than $\Omega_{M}$ or $\Omega_{\Lambda}$. A possible observable is the average of $\cos \left(\theta_{\mathrm{GAL}}\right)$ over the total survey area:

$$
\left\langle\cos \left(\theta_{\mathrm{GAL}}\right)\right\rangle=\frac{3}{2} \sqrt{\frac{\pi}{2} \frac{\left\langle\kappa \kappa_{\mathrm{GAL}}\right\rangle}{\left\langle\kappa_{\mathrm{GAL}}^{2}\right\rangle} .}
$$

If the CMB ellipticities are significantly aligned with the distant galaxies, then $\left\langle\cos \left(\theta_{\mathrm{GAL}}\right)\right\rangle$ should be significantly larger than zero. Some values of $\left\langle\cos \left(\theta_{\mathrm{GAL}}\right)\right\rangle$ are given in Table 3 for various cosmological models and smoothing schemes.

This table also shows the estimates of the cosmic variance of such detection and it is found to be rather modest. Even with a full sky cosmic survey, that would be the case. A detection might be possible but the amplitude will be poorly determined. Clearly these results call for a search of such effects in the polarization field.

\subsection{From the polarization field}

The magnetic component of the polarization in eq. (2.8) appears to be built from a pure CMB part, which comes from the primordial polarization, and a gravitational lensing part. It is natural to define $b$, in such a way that mimics the $\Delta \hat{B}$ function dependence, by replacing the $\mathrm{CMB}$ shear field by the galaxy one.

$$
\begin{aligned}
b_{\mathrm{GAL}} & =\epsilon_{i j}\left(\gamma_{\mathrm{GAL}}^{i} \Delta \hat{P}^{j}+\gamma_{\mathrm{GAL}, k}^{i} \hat{P}^{j, k}\right) \\
& =\epsilon_{i j}\left(\gamma_{\mathrm{GAL}}^{i} \Delta P^{j}+\gamma_{\mathrm{GAL}, k}^{i} P^{j, k}\right)+O\left(\kappa^{2}\right) .
\end{aligned}
$$




\begin{tabular}{|c|c|c|c|c|}
\hline & \multicolumn{2}{|c|}{ Cosmic Variance $\left(\mathcal{X}_{1}\right)$} & \multicolumn{2}{|c|}{ Cosmic Variance $(\mathcal{X}$} \\
\hline & $\Omega_{0}=0.3$ & $\Omega_{0}=1$ & $\Omega_{0}=0.3$ & $\Omega_{0}=1$ \\
\hline$\theta=5^{\prime}, \theta_{\mathrm{GAL}}=2.5^{\prime}$ & $6.44 \%$ & $4.77 \%$ & $6.06 \%$ & $4.72 \%$ \\
\hline$\theta=5^{\prime}, \theta_{\mathrm{GAL}}=5^{\prime}$ & $6.58 \%$ & $4.79 \%$ & $4.99 \%$ & $4.23 \%$ \\
\hline$\theta=10^{\prime}, \theta_{\mathrm{GAL}}=5^{\prime}$ & $8.71 \%$ & $6.73 \%$ & $9.49 \%$ & $7.62 \%$ \\
\hline
\end{tabular}

Table 4. Values of the cosmic variance of $\mathcal{X}$ (the two columns correspond to the two terms that appear in 5.5) for a survey size of $100 \mathrm{deg}^{2}$.

This new quantity can be viewed as a guess for the CMB polarization $B$ component if lensing was turned on only in a redshift range matching the depth of galaxy surveys. The correlation coefficient of this guess with the true $\Delta B$ field, that is $\left\langle\Delta \hat{B} b_{\mathrm{GAL}}\right\rangle$, is expected to be quadratic both in $P$ and in $\gamma$ and to be proportional to the cross-coefficient $r$.

The key issue of this construction is not so much to show that $b_{\mathrm{GAL}}$ and $B$ are indeed cross-correlated (which by construction is bound to happen) but that the cosmic variance of the cross-correlation signal is large enough. Actually the cosmic variance of such a quantity can be investigated in a large part analytically. This has been done in Benabed et al. (2001).

The biggest source of error is the measure of $\left\langle\Delta \hat{B} b_{\mathrm{GAL}}\right\rangle$. It is given by,

$$
C_{\mathrm{GAL}}=\sqrt{\left\langle\left(\overline{\Delta \hat{B} b_{\mathrm{GAL}}}-\overline{\Delta \hat{B}} \overline{b_{\mathrm{GAL}}}\right)^{2}\right\rangle-\left\langle\overline{\Delta \hat{B} b_{\mathrm{GAL}}}-\overline{\Delta \hat{B}} \overline{b_{\mathrm{GAL}}}\right\rangle^{2}}
$$

where overlined quantities stand for geometrical averages (e.g. average over the survey in which the measurement is made). Indeed we can neglect the errors on $\left\langle\Delta \hat{E}^{2}\right\rangle,\left\langle(\nabla \hat{E})^{2}\right\rangle$, $\left\langle\left(\nabla \kappa_{\mathrm{GAL}}\right)^{2}\right\rangle$ and $\left\langle\kappa_{\mathrm{GAL}}^{2}\right\rangle$; those may not be the dominant source of discrepancy and can even be measured on wider and independent samples. The terms that appear in such expressions can be estimated one by one if we assume that all the involved fields follow Gaussian statistics, which is reasonable for the scales we are working on. In that case indeed, we can take advantage of the Wick theorem to contract each of the 8 fields correlators in products of 2 points correlation functions. By definition, (5.6) contains only connected correlators, moreover the ensemble averages $\langle\Delta \hat{B}\rangle$ and $\left\langle b_{\sharp}\right\rangle$ vanish, therefore only a small fraction of correlators among all the possible combination of the 8 fields survive. We can use a simple diagrammatic representations to describe their geometrical shape, Fig. 11. Obviously if the survey is large enough and since the lens effect is only a small perturbation effect, the $n$-points correlation functions naturally dominates over the $n+1$-points correlation function. This is true as long as the local variance is much bigger than the autocorrelation at survey scale and we assume the surveys are still large enough to be in this case. Then the $\mathcal{A}$ terms n Fig. 11 dominate over the others.

Eventually the resulting cosmic variance can be estimated from semi-analytic method. The results are presented in Table 4 .

Clearly, and contrary to what is obtained when the temperature maps is used alone, such a correlation can potentially be measured with a rather high signal to noise ratio even in surveys of rather modest size and resolution. Anticipating data sets that should be available in the near future, $\left(100 \mathrm{deg}^{2}\right.$ survey, with $5^{\prime}$ resolution for galaxy survey and $10^{\prime}$ Gaussian beam size for CMB polarization detection), we have obtained a cosmic variance around $8 \%$. Needless is to say that this estimation does not take into account systematics and possible foreground contaminations. 

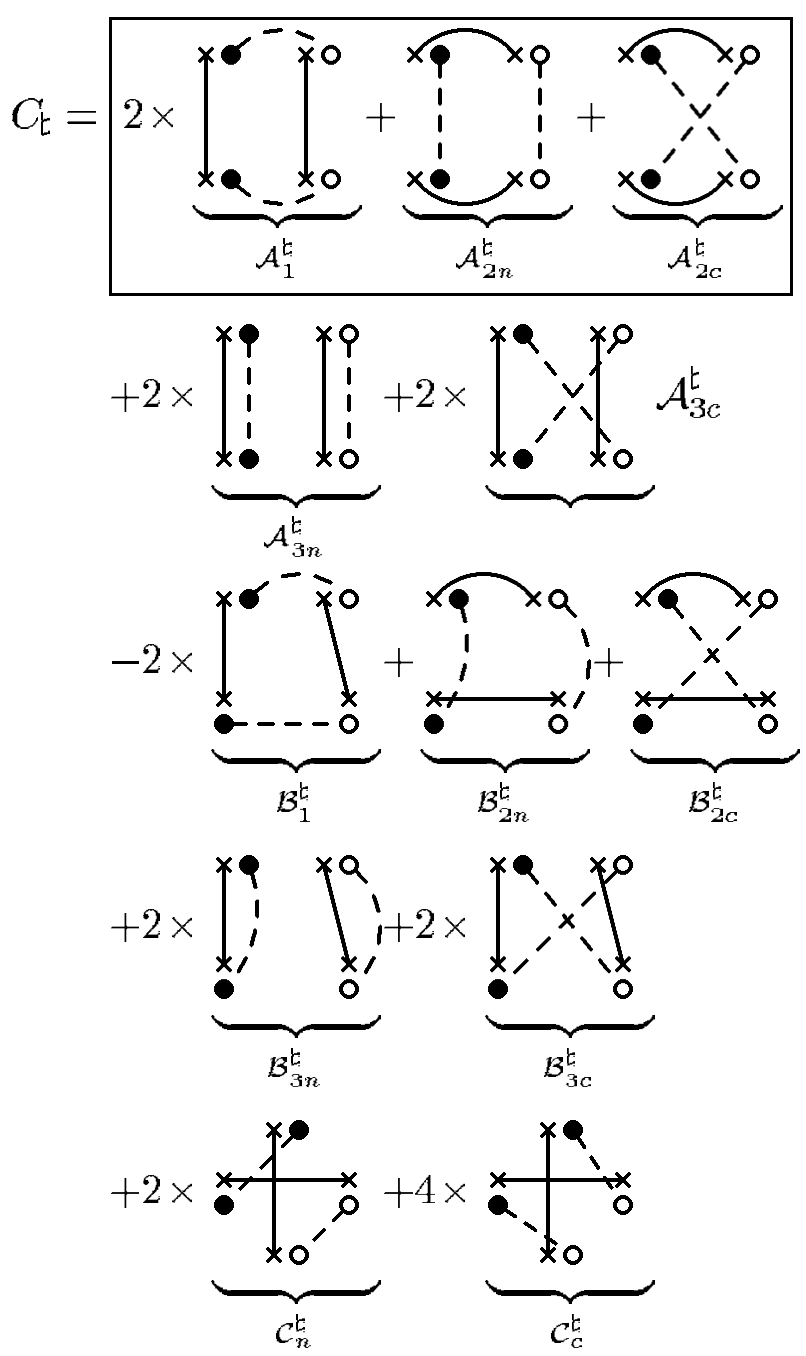

Figure 11. Diagrammatic representation of the terms contributing to the cosmic variance of the correlation coefficients. In this representation the vertex $\times \bullet$ represents $\Delta \hat{B}$; the cross stands for the $\Delta P$ part, the dot for $\gamma_{\mathrm{CMB}}$. The other vertex $\times o$ represents any one of the two terms in $b_{\mathrm{GAL}}$; the open dot stands for $\gamma_{\mathrm{GAL}}$. The solid lines connect $\Delta P$ terms and the dashed ones the $\gamma$-s

\subsection{Further consequences}

The detection of cross-correlation effects between CMB observations and large-scale structure observations is a very attractive domain of research. It is natural to try to correlate cosmic shear survey with CMB data because the former is a direct tracer of the potential wells that are responsible for the CMB lens effects. However any tracer of the large-scale structure is bound to be correlated with CMB anisotropies and polarization!

For instance it has been noticed that correlation with ISW or SZ induces 3-pt correlation functions ( Goldberg \& Spergel 1999, Cooray \& Hu 2000) in the temperature maps.

Largescale structure is also traced by dusty starforming galaxies, that induce anisotropies in the farinfrared background (FIRB) (Song et al. 02). 


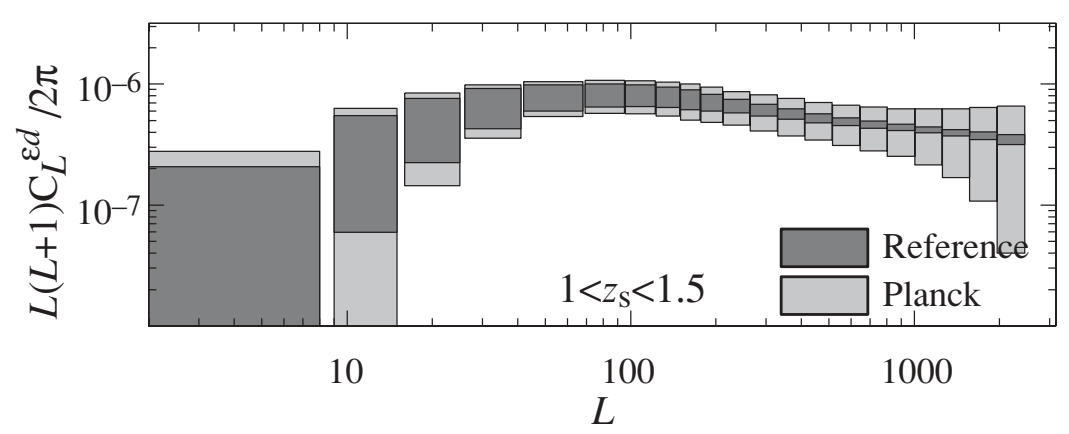

Figure 12. Statistical errors on the cross correlation of CMB deflections and cosmic shear in three source redshift bands on a $1000 \mathrm{deg}^{2}$ patch of sky for the Planck and reference experiment.

It is to be noted however that other mechanisms might be responsible for other CMBLSS correlation patterns. They are the ISW effect at large scale due to the time variation of the linear potentials in non EdS universe (large angular scales) an the thermal and kinetic SZ effects from clusters. The latter is probably the main source of worries for accurate reconstructions of the convergence maps Amblard, Vale \& White 2004.

\subsection{What would we learn?}

Probably the best reason for which one wants to try to detect CMB lensing effects is that it should be there if the gravitational instability picture, upon which our current understanding of cosmology is based, is correct. Lensing effects, because it is totally independent of further modelling, provides us with a very solid consistency check of the theory.

Moreover, the detection of lens effects on the last scattering surface would provide us with precious new constraints on the cosmological parameters. One comes from the overall amplitude of the effect which is directly sensitive to the cosmological parameters: e.g. the effect is all the more important that the optical bench in which it takes place is deep.

Probably more interesting are the constraints that tomographic view of the dark matter distribution can give. This possibility is illustrated on Fig. 12 which exhibits the expected cross-spectra between galaxy lensing and CMB lensing observations. From such observations the growth rate history of the fluctuation should be accessible to observations giving precious new constraints on the cosmological background and in particular on the dark energy equation of state. With such observation one also could try to design tests of the large-scale gravity laws. After all the Poisson equation has never been tested for such cosmological scales!

\section{Conclusions}

CMB lensing has been examined extensively over the last few years. The signatures of such effects are to be found in the non-Gaussian properties they induce. The computation of the four-point functions has given birth to methods for measuring lens effects in CMB maps. Reconstruction methods of the displacement field are now established on solid grounds, at least from a theoretical point of view, although further numerical studies are needed (see Amblard, Vale \& White 2004 and this proceedings). So far no detection of cross-correlation has been established. Attempts with the WMAP data have not produced any significant results (see Hirata et al. 2004 and this proceedings.) 
It is also to be remarked that lens effects have specific correlation patterns that should be clearly identified and not be misinterpreted as primordial Non-Gaussian effects.

Reconstruction methods of the lens field acting on the CMB, together with galaxy lensing, open the way to tomographic exploration of the dark matter distribution of the Universe. There are probably numerous data analysis issues to be solved to succeed in doing so. The Planck satellite and the galaxy lensing surveys that are now in progress should provide us with data sets large enough to experiment these methods. But space imaging is probably what we need to get the scientific reward these phenomena promise. This is indeed probably one of our best chances to uncover the nature of the dark energy, at least to infer how close (or not) to a cosmological constant this mysterious component of our universe is.

\section{Acknowledgements}

I would like to thank the organizers of the conference for inviting me to give this review talk. It gave me the opportunity to appreciate how much this field has grown in the past few years!

\section{References}

Amblard, A, Vale, C. \& White, M. 2004, astro-ph/0403075

Benabed, K. \& Bernardeau, F. 2000, Phys. Rev. D 61, 123510

Benabed, K., Bernardeau, F., van Waerbeke, L. 2001, Phys. Rev. D 63, 043501

Bernardeau, F. 1997, Astron. \& Astrophys. 324, 15-26.

Bernardeau, F. 1998, Astron. \& Astrophys. 338, 375-382

Bond, J.R. \& Efstathiou, G. 1987, Month. Not. of the R.A.S. 226, 655-687.

Cooray, A. \& Hu, W. 2000, Astrophys. J. 534, 533-550

Faraoni, V. 1993, Astron. \&3 Astrophys. 272, 385-388

Goldberg, D. M. \& Spergel, D.N. 1999, Phys. Rev. D 59, 103002

Hirata, C. M. \& Seljak, U. 2003, Phys. Rev. D 67, 43001

Hirata, C. M. \& Seljak, U. 2003, Phys. Rev. D 68, 83002

Hirata, C. M. Padmanabhan, N., Seljak, U., Schlegel, D., Brinkmann, J. 2004, submitted to $P R D$, astro-ph/0406004

Hu, W. 2000 Phys. Rev. D 62043007

Hu, W. \& Okamoto, T. 2002, Astrophys. J. 574, 566-574

Kesden, M., Cooray, A. \& Kamionkowski, M. 2002, Phys. Rev. D 66, 083007

Kesden, M., Cooray, A., Kamionkowski, M. 2003, Phys. Rev. D 67, 123507

Mellier, Y. 1999, Annual Review of Astron. and Astrophys., 37, 127-189

Okamoto, T. \& Hu, W. 2002, Phys. Rev. D 66, 063008

Peacock, J.A. \& Dodds, S.J. 1996, MNRAS, 280, L19-L26

Seljak, U. 1996, Astrophys. J. 463 1-7

Schmalzing, J., Takada, M. \& Futamase, T. 2000, Astrophys. J., 544, L83-L86

Song, Y.-S., Cooray, A., Knox, L. \& Zaldarriaga, M. 2003, Astrophys. J. 590, 664-672

Takada, M. 2001, Astrophys. J., 558, 29-41

Takada, M., Komatsu, E. \& Futamase, T. 2000, Astrophys. J., 533, L83-L87

Van Waerbeke, L., Bernardeau, F., Benabed, K. 2000 Astrophys. J. 540 14-19

Zaldarriaga, M. \& Seljak, U. 1998, Phys.Rev. D58 023003 